\title{
Guest Editorial: Image Analysis and Processing Leveraging Additional Information
}

\author{
Luis Herranz ${ }^{1} \cdot$ Jian Cheng $^{2}$ - Yue Gao ${ }^{3}$. \\ Shuqiang Jiang ${ }^{1}$
}

Published online: 16 March 2016

(C) Springer Science+Business Media New York 2016

Many multimedia analysis and processing applications can benefit from better image understanding. However, accurate understanding of what the underlying visual content represents still remains a very challenging problem. Systems need to look beyond the pure matrix of intensity values and exploit other types of information. Actually, humans do not solve visual problems based just on the captured pixel data. Many non-visual clues and diverse types of external information are exploited, including prior knowledge, prior experience, contextual and collaborative information. Intelligent systems can often incorporate them to simplify the problem, and can also integrate other types of signals, such depth and infrarred images. Often this additional information can be critical to better address the problem or increase the quality of the result. This special issue contains 10 papers, selected from the inital 25 submissions, after two rounds of blind review.

Priveleged or expert information can help to guide learning by exploiting indirect cues. In "Facial Expression Recognition through Modeling Age-related Spatial Patterns" [6], Wang et al describe a system that leverages age information, available only during training, to

Luis Herranz

luis.herranz@vipl.ict.ac.cn

Jian Cheng

jcheng@nlpr.ia.ac.cn

Yue Gao

kevin.gaoy@gmail.com

Shuqiang Jiang

sqjiang@ict.ac.cn

1 Key Laboratory of Intelligent Information Processing, Institute of Computing Technology, Chinese Academy of Sciences, Beijing 100190, China

2 National Laboratory of Pattern Recognition, Institute of Automation, Chinese Academy of Sciences, Beijing 100190, China

3 School of Software, Tsinghua University, Beijing, 100086 China 
improve facial expression recognition. Age-related spatial expression patterns carry crucial information that has not been exploited previously. In particular, the authors implement this system using Bayesian networks modeling facial geometric features, outperforming existing approaches. The paper "Maximum Margin Hashing with Supervised Information" [8], by Yang et al, describes a large margin approach to learn hashing functions in a supervised way. Leveraging label information helps to obtain hashing functions that better represent the semantic similarity between images. The paper "Discriminative Sparse Representation for Face Recognition" [10], by Zhang et al, proposes to use facial structure information to increase face recognition performance. The weights are learned for different face locations based on information entropy, providing an effective prior and structure information. A sparse coding formulation combined with this weighting scheme results in more discriminative representations. Experiments on face recognition benchmarks show good results. In "High-Order Graph Matching Kernel for Early Carcinoma EUS Image Classification" [9], Zhang et al address the problem of early carcinoma detection in electronic ultrasonography images. The method is based on graph matching, and the authors propose a new kernel that preserves better the underlying topological structure of the graph. The paper "Discriminative Sparse Neighbor Coding" [1], by Bai et al, proposes a new coding method addressing some shortcomings of traditional sparse coding strategies. They include a module that selects discriminative features for each class, and then another module that discards non-informative visual words. Finally they obtain class-specific low dimensional subspaces, with results achieving state-of-the-art performance.

Combining multiple heterogeneous signals or multiple features extracted from the same signal can also outperform methods using a single one. In "Features combination for art authentication studies: brushstroke and materials analysis of Amadeo de Souza-Cardoso" [4], Montagner et al tackle the interesting problem of art authentication, in the particular case of the modernist painter Amadeo de Souza-Cardoso. In addition to conventional RGB analysis, which is used to analyze brushstroke characteristics, their system incorporates hyperspectral imaging and elemental analysis to determine the pigments present in the painting and compare them with those used by the painter. "Camouflage Performance Analysis and Evaluation Framework Based on Features Fusion" [7], by Xue et al, proposes a model to evaluate the quality of camouflage features. Their model uses nonlinear fusion to combine multiple image features that measure the degree to which the target and surrounding background differ with respect to background-related and internal features. Subjective experiments show that scores predicted by the system correlate with the difficulty human assessors had to detect camouflaged targets. In "Pointwise and Pairwise Clothing Annotation: Combining Features from Social Media" [5], Nogueira et al exploit social media data to automatically annotate clothes, including the different garment items. The authors formulate the task as a multi-label and multi-modal classification problem, proposing two approaches (pointwise and pairwise). Their experiments show a significant improvement compared with related methods.

External information can be used in image enhancement and other image processing operations. The paper "Image Super-Resolution based on Multi-kernel Regression", by Li [2] et al, describes a data-driven method to obtain high resolution images from blurred ones using multi-kernel regression, and an efficient algorithm to implement it. The key information to obtain the details that enhance the images is learned from an external dataset. In "LWT- QR decomposition-based robust and efficient image watermarking scheme using Lagrangian SVR" [3], Mehta et al address the problem of watermaking images, that is, embedding an image with an external signal in a such a way that the signal cannot be perceived, but recovered if necessary. Their method uses the coefficients from a lifting 
transform followed by the $\mathrm{QR}$ decomposition to train a support vector regression model. The model is used to embed the watermark and then to extract it when necessary.

These ten papers cover a wide range of image analysis and processing applications and scenarios in which additional information is beneficial. We hope the readers will find interesting ideas in them. Finally, to conclude, the guest editors would like to express their gratitude to the authors who submitted manuscripts and to the reviewers who generously contributed reviewing them.

\section{References}

1. Bai X, Yan C, Ren P, Bai L, Zhou J (2015) Discriminative sparse neighbor coding. Multimed Tools Appl. doi:10.1007/s11042-015-2951-4

2. Li J, Qu Y, Li C, Xie Y (2015) Image super-resolution base on multi-kernel regression. Multimed Tools Appl doi:10.1007/s11042-015-3016-4

3. Mehta R, Rajpal N, Vishwakarma VP (2015) Lwt- qr decomposition based robust and efficient image watermarking scheme using lagrangian svr. Multimed Tools Appl doi:10.1007/s11042-015-3084-5

4. Montagner C, Jesus R, Correia N, Vilarigues M, Macedo R, Melo MJ (2016) Features combination for art authentication studies: Brushstroke and materials analysis of amadeo de souza-cardoso. Multimed Tools Appl doi:10.1007/s11042-015-3197-x

5. Nogueira K, Veloso, Santos JA (2015) Pointwise and pairwise clothing annotation: combining features from social media. Multimed Tools Appl doi:10.1007/s11042-015-3087-2

6. Wang S, Wu S, Gao Z, Ji Q (2015) Facial expression recognition through modeling age-related spatial patterns. Multimed Tools Appl doi:10.1007/s11042-015-3107-2

7. Xue F, Yong C, Xu S, Dong H, Luo Y, Jia W (2015) Camouflage performance analysis and evaluation framework based on features fusion. Multimed Tools Appl doi:10.1007/s11042-015-2946-1

8. Yang H, Bai X, Liu Y, Wang Y, Bai L, Zhou J, Tang W (2016) Maximum margin hashing with supervised information. Multimed Tools Appl doi:10.1007/s11042-015-3159-3

9. Zhang Z, Bai L, Ren P, Hancock ER (2015) High-order graph matching kernel for early carcinoma eus image classification. Multimed Tools Appl doi:10.1007/s11042-015-3108-1

10. Zhang Z, Liang Y, Bai L, Hancock ER (2015) Discriminative sparse representation for face recognition. Multimed Tools Appl doi:10.1007/s11042-015-3136-X

Luis Herranz received the Ph.D in Computer Science and Telecommunication from the Universidad Autónoma de Madrid, Spain in 2010. From 2003 to 2010, he was with the Escuela Politécnica Superior of the Universidad Autónoma de Madrid as a researcher and teaching assistant. From 2010 to 2011 he was with Mitsubishi Electric R\&D Centre Europe, United Kingdom. He is currently a postdoctoral research fellow with the Institute of Computing Technology of the Chinese Academy of Sciences, Beijing, China. His research interests include image understanding, computer vision, machine learning and multimedia indexing and retrieval.

Jian Cheng is currently a professor of Institute of Automation, Chinese Academy of Sciences. He received the B.S. and M.S. degrees in Mathematics from Wuhan University in 1998 and in 2001, respectively. In 2004, he got his Ph.D degree from Institute of Automation, Chinese Academy of Sciences. His current research interests include image and video search, machine learning, data mining, etc. He has authored or co-authored more than 100 academic papers and co-edited two books in these areas. He was the recipient of LU JIAXi Young Talent award in 2010. Prof. Cheng served as Program co-chair for ACM International Conference on Internet Multimedia Computing and Services (ICIMCS10), Technical Program Committee member for ACM Multimedia 2008-2009, IEEE Conference on Computer Vision and Pattern Recognition (CVPR08), IEEE International Conference on Multimedia and Expo (ICME08-12), IEEE International Conference on Computer Vision (ICCV07), etc.

Yue Gao received the B.S. degree from the Harbin Institute of Technology, Harbin, China, and the M.E. and Ph.D. degrees from Tsinghua University, Beijing, China. 
Shuqiang Jiang is a professor with the Institute of Computing Technology, Chinese Academy of Sciences, Beijing. He is also with the Key Laboratory of Intelligent Information Processing, Chinese Academy of Sciences. His research interests include multimedia processing and semantic understanding, pattern recognition, and computer vision. He has authored or coauthored more than 100 papers on the related research topics. Dr. Jiang was supported by the New-Star program of Science and Technology of Beijing Metropolis in 2008. He won the Lu Jiaxi Young Talent Award from Chinese Academy of Sciences in 2012, and the CCF Award of Science and Technology in 2012. He is the senior member of IEEE, member of ACM, CCF, and YOCSEF. Prof. Jiang is the executive committee member of ACM SIGMM China chapter. He has been serving as the guest editor of the special issues for PR and MTA. He is the program chair of ICIMCS2010, special session chair of PCM2008, ICIMCS2012, area chair of PCIVT2011, publicity chair of PCM2011 and proceedings chair of MMSP2011. He has also served as a TPC member for more than 20 well-known conferences, including ACM Multimedia, CVPR, ICCV, ICME, ICIP, and PCM. 